\title{
La competitividad y sus factores determinantes: un análisis sistémico para países en desarrollo
}

\author{
Victor Medeiros, Lucas Gonçalves Godoi \\ y Evandro Camargos Teixeira
}

\section{Resumen}

En este trabajo se presenta un análisis comparativo y econométrico de la competitividad en los países en desarrollo y se indican sus determinantes y fuentes de variación. Se utiliza la metodología de análisis envolvente de datos para generar medidas de competitividad. Se obtienen los determinantes de la competitividad mediante el empleo de un modelo Tobit sobre la base del enfoque de competitividad sistémica. Visto el bajo nivel medio de competitividad, los principales resultados indican que gran parte de los países de la muestra mejoren en términos de asignación de recursos. Además de factores empresariales como la innovación y la sofisticación del ambiente de negocios, algunos aspectos estructurales, como el tamaño del mercado y la calidad de la demanda, y factores sistémicos como la infraestructura, la salud, la educación, la capacitación de la mano de obra y el ambiente macroeconómico, son condiciones importantes para aumentar la competitividad de los países emergentes.

\section{Palabras clave}

Crecimiento económico, competitividad, productividad, eficacia industrial, países en desarrollo, análisis comparativo, modelos econométricos

\section{Clasificación JEL}

C24, O20, 057

\section{Autores}

Victor Medeiros es doctorando en economía en la Facultad de Ciencias Económicas del Centro de Desarrollo y Planificación Regional de la Universidad Federal de Minas Gerais, Brasil. Correo electrónico: victor-medeiros@cedeplar.ufmg.br.

Lucas Gonçalves Godoi es titular de una maestría en economía de la Facultad de Economía, Administración y Contabilidad de Ribeirão Preto de la Universidad de São Paulo, Brasil. Correo electrónico: lucasgodoi@usp.br.

Evandro Camargos Teixeira es profesor adjunto en el Departamento de Economía de la Universidad Federal de Viçosa, Brasil. Correo electrónico: evandro.teixeira@ufv.br. 


\section{Introducción}

De acuerdo con Ferraz, Kupfer y Haguenauer (1996), la competitividad puede definirse como la capacidad de una empresa para crear e implementar estrategias competitivas y mantener o aumentar su cuota de productos en el mercado de manera sostenible. Esas capacidades están relacionadas con diversos factores, controlados o no por las empresas, que van desde la capacitación técnica del personal y los procesos gerenciales-administrativos hasta las políticas públicas, la oferta de infraestructura y las peculiaridades de la demanda y la oferta.

La importancia de la competitividad puede observarse en su relación positiva con el crecimiento económico a largo plazo. Un país puede considerarse competitivo cuando logra manejar sus recursos y competencias de manera que, además de aumentar la producción de sus empresas, mejora la calidad de vida de sus ciudadanos (IMD, 2012). De acuerdo con una definición estándar de la Unión Europea (2001), la competitividad, a nivel regional y nacional, es la capacidad de un determinado país o región de generar mayores tasas de crecimiento y empleo de manera sostenible.

Algunos autores investigaron los principales determinantes de la competitividad y su relación con el proceso de crecimiento y desarrollo económico de los países. En los diversos estudios que componen la vertiente teórica, los principales determinantes de la competitividad $-\mathrm{y}$, en consecuencia, del crecimiento económico - incluyen, entre otros: las inversiones en capital, la división del trabajo y el comercio, según la teoría clásica; la intensidad de capital, las inversiones y las políticas gubernamentales de subsidios y tasas, en el enfoque keynesiano; el cambio estructural, la apertura comercial y la inversión extranjera directa, desde el punto de vista de la economía del desarrollo; y el nivel educativo, el gasto en capital humano, investigación y desarrollo $(I+D)$ e incentivos a la innovación, en la "nueva teoría del crecimiento"1.

Además, los teóricos del crecimiento endógeno destacaron aspectos como el capital humano (Lucas, 1988), las innovaciones (Romer, 1990; Aghion y Howitt, 1992), la infraestructura (Barro, 1990), las instituciones (Romer, 1986) y la competencia y la apertura comercial (Grosmman y Helpman, 1991).

De manera similar, Ferraz, Kupfer y Haguenauer (1996) elaboraron un enfoque de la competitividad como proceso dinámico, es decir, la competitividad de un país está determinada y limitada por una diversidad de factores empresariales, estructurales y sistémicos. En este enfoque dinámico, las ventajas competitivas nacen de las variables de relevancia competitiva en todos los niveles del sistema y de la interacción entre ellas. Así, se concluye que la competitividad tiene un carácter sistémico (Esser y otros, 1996).

La evidencia empírica ha corroborado los estudios teóricos mencionados anteriormente. Aunque esta literatura no es vasta, se ha demostrado la hipótesis de una relación positiva entre competitividad y crecimiento económico, como, por ejemplo, en la investigación de Kordalska y Olcyzk (2016) $)^{2}$.

Con el uso de la metodología de análisis envolvente de datos (DEA) surgieron otros estudios para determinar el nivel de competitividad de los países y sus factores determinantes mediante un análisis comparativo (Rocha, Rebelatto y Camioto, 2015; Ülengin y otros, 2011; Charles y Zegarra, 2014). En estos casos, la competitividad se trató en un concepto ex ante, es decir, se consideraría competitivo al país que asignara sus recursos de manera eficiente con respecto a los demás. Entre los principales resultados obtenidos, se destaca que factores como la educación, la geografía, la igualdad de ingresos, las buenas instituciones y la provisión de bienes públicos pueden ser importantes para que los países utilicen sus recursos de manera eficiente y, en consecuencia, sean más competitivos.

\footnotetext{
1 Véanse más detalles en Kordalska y Olcyzk (2016).

2 En este caso se utilizó un modelo de datos de panel.
} 
Con el presente artículo se desea contribuir a la literatura especializada en la competitividad al brindar más evidencia sobre sus principales determinantes en los países en desarrollo, mediante un análisis comparativo y econométrico para el período 2011-2014, pues no se han encontrado estudios en los que se relacionen estos dos aspectos. La elección de los países emergentes se justifica por su rezago competitivo con respecto al resto del mundo (observado, por ejemplo, en el Índice de Competitividad Global (ICG)), pues la mejora de los aspectos que aumentan la competitividad de los países podría ser un factor importante para promover el crecimiento económico sostenido en esas naciones ${ }^{3}$.

Para lograr los objetivos de este trabajo se utilizará la metodología DEA, a fin de generar las medidas de eficiencia de los países a partir de una función de producción agregada. Estas medidas servirán como variable sustitutiva de la competitividad comparativa nacional. Asimismo, por medio del índice de Malmquist, se procurará obtener las variaciones en la productividad total de los factores de producción (PTF) y su descomposición en factores de cambio (cambios técnicos y en la eficiencia técnica) ${ }^{4}$. Posteriormente, se estimará un modelo econométrico para establecer los factores determinantes de la competitividad, con el fin de brindar más evidencia sobre las posibles políticas que se han de implementar en los países de la muestra para aumentar la competitividad y continuar el proceso de crecimiento económico. Los factores determinantes, a su vez, se establecerán a partir del análisis de componentes principales, debido al alto grado de multicolinealidad entre las variables involucradas.

El trabajo se divide en cinco secciones, incluida esta introducción. Mientras en la segunda se aborda el marco teórico, en la tercera se presentan los métodos utilizados en el artículo. En la cuarta sección se describen los resultados obtenidos y en la quinta y última se resumen las principales conclusiones.

\section{Crecimiento, competitividad y sus factores determinantes: un enfoque sistémico}

En la literatura, la competitividad se define mediante diversos conceptos e indicadores. En el concepto de desempeño, la competitividad está relacionada con el rendimiento de las exportaciones industriales del país. Según este concepto ex post, las empresas son competitivas cuando logran ampliar su participación en el mercado internacional de determinados productos (Haguenauer, 1989). Además de las condiciones de producción, la competitividad a través del desempeño comprende los factores que estimulan o desalientan las exportaciones de productos y países específicos, las políticas cambiaria y comercial, la eficiencia de los canales de comercialización y de los sistemas de financiamiento, los acuerdos internacionales y las estrategias de las empresas.

Para otra línea de autores, el desempeño de las exportaciones sería una consecuencia inmediata de la competitividad y no al contrario. El concepto en este punto de vista es potencial, ex ante, y tradicionalmente está ligado a las condiciones de producción. Según el concepto de eficiencia, la competitividad se define como la capacidad de un país de producir un determinado bien en mejores o iguales condiciones en comparación con otras economías (Haguenauer, 1989).

\footnotetext{
3 La elección se justifica, además, por otras características similares de los países analizados, como menores niveles de ingreso per cápita con respecto a los países desarrollados, un modelo de exportación compuesto mayoritariamente por productos con menor contenido tecnológico y peores indicadores de desarrollo humano. Además, en aspectos metodológicos, se asume que dichos países tienen características similares entre ellos, que vuelven más realista el análisis a partir del método DEA y su enfoque comparativo.

4 Entre los autores que utilizaron esta metodología aplicada a la evaluación de la productividad total de los factores para países en desarrollo se pueden citar Marinho y Bittencourt (2007) y Araujo, Feitosa y Da Silva (2014).
} 
En el enfoque de eficiencia, se considera competitivo al país que logra generar el mayor producto, en comparación con los demás, dados los recursos de que dispone. De esta forma, se puede considerar una función de producción agregada simple, descrita por:

$$
Y=A K^{\alpha} L^{1-\alpha}
$$

En que $Y$ es el producto, $K$ el nivel de capital, $L$ el número de trabajadores y $\alpha$ un parámetro positivo, entre 0 y 1 , que describe el rendimiento del factor capital. Se consideraría eficiente al país que, manteniendo la tecnología constante, utiliza sus insumos de capital y trabajo de manera de generar el mayor producto posible. En este caso, el país estaría en la frontera de la tecnología de producción, de forma que no se desperdiciarían sus factores productivos y el nivel de ingresos sería el más alto posible para su población.

En este estudio se utiliza el concepto de competitividad como eficiencia, pues su objetivo consiste en determinar la eficiencia relativa de los países en desarrollo. Sin embargo, como esta medida no basta para describir la magnitud del término competitividad, es necesario analizar también los factores que la determinan, de acuerdo con el enfoque de Ferraz, Kupfer y Haguenauer (1996). Estos autores buscan un enfoque dinámico, que proporcione un punto de referencia para la competitividad mediante el análisis del proceso competitivo. En este contexto, la competitividad se define como la capacidad de una empresa de crear e implementar estrategias competitivas y mantener o aumentar su cuota de mercado de manera sostenible.

El desempeño y la eficiencia productiva de las empresas estarían ligados a la capacitación acumulada a lo largo del tiempo, que incidiría en sus estrategias competitivas. Estas, a su vez, se adoptarían de acuerdo con el ambiente económico y el proceso competitivo en el cual se insertaría la empresa. De esta forma, la competitividad se entendería como una medida que abarca no solo una determinada empresa, sino que está directamente relacionada con el patrón de competencia de un mercado específico.

Los patrones de competencia estarían influenciados por la composición del ambiente competitivo de la empresa, es decir, por los componentes estructurales y conductuales de un determinado sector o sistema económico. Estos patrones derivan de la interdependencia entre las empresas o sectores competitivos a través de complementariedades tecnológicas, restricciones y estímulos al flujo de bienes, además de la disponibilidad de infraestructura, leyes, sistemas de planificación y políticas industriales, entre otros. La competitividad sería la capacidad de adaptación de las estrategias de las empresas al patrón de competencia vigente en el ramo en que actúan.

Así, el análisis de la competitividad debe tener en cuenta la diversidad de factores (procesos de esfuerzo de ventas, capacitación productiva, acceso a fuentes de materias primas y proveedores de piezas y componentes, además de los directamente vinculados con la innovación y la difusión de nuevas técnicas) que son capaces de generar ventajas competitivas (Ferraz, Kupfer y Haguenauer, 1996). De esta forma, se crea un enfoque dinámico del desempeño competitivo al incorporar sus factores determinantes de naturaleza empresarial, estructural y sistémica. En otras palabras, la competitividad es, en esencia, sistémica (Esser y otros, 1996).

Los "factores empresariales" son aquellos sobre los cuales la empresa tiene un control sustancial. Estos factores se relacionan con la acumulación de conocimiento generado por la empresa y sus estrategias, y, más específicamente, con la eficacia de la gestión, la capacitación y el desempeño, la capacitación tecnológica en procesos y productos, los métodos de organización y los recursos humanos, entre otros. 
Los "factores estructurales" son aquellos sobre los cuales la empresa no tiene pleno control, es decir, las empresas están limitadas por el propio proceso de competencia y sus especificidades. Además de las características de la demanda y la oferta, estos incluyen la influencia de instituciones fuera del mercado que definen el régimen de incentivos y regulación, la distribución geográfica, el grado de sofisticación tecnológica, las tasas de crecimiento, los sistemas de comercialización y las oportunidades de acceso a productos internacionales, entre otros (Ferraz, Kupfer y Haguenauer, 1996).

Los "factores sistémicos" son aquellos sobre los cuales el control de la empresa es muy escaso o nulo. Estos factores son responsables de generar externalidades a las empresas, actuando como parámetros del proceso de decisión. Entre estos factores se destacan los siguientes: infraestructurales (disponibilidad, calidad y costo de la energía, transporte, telecomunicaciones, insumos básicos y servicios tecnológicos); macroeconómicos (tipo de cambio, carga tributaria, tasa de crecimiento del producto interno, oferta de crédito y tasas de interés y política salarial); político-institucionales (política tributaria, política arancelaria, apoyo fiscal al riesgo tecnológico, poder adquisitivo del gobierno); sociales (sistema de calificación de la mano de obra, políticas de educación y formación de recursos humanos, laboral y de seguridad social); legales y normativos (políticas de protección de la propiedad industrial, preservación del medio ambiente, defensa de la competencia y protección del consumidor); e internacionales (tendencias del comercio mundial, flujos internacionales de capital, inversiones de riesgo y tecnología, relaciones con organismos multilaterales).

En el diagrama 1 se muestra el alcance del término competitividad, en el que la empresa constituye el elemento central, que a su vez resulta afectado por la estructura y el sistema económico.

Diagrama 1

Factores determinantes de la competitividad

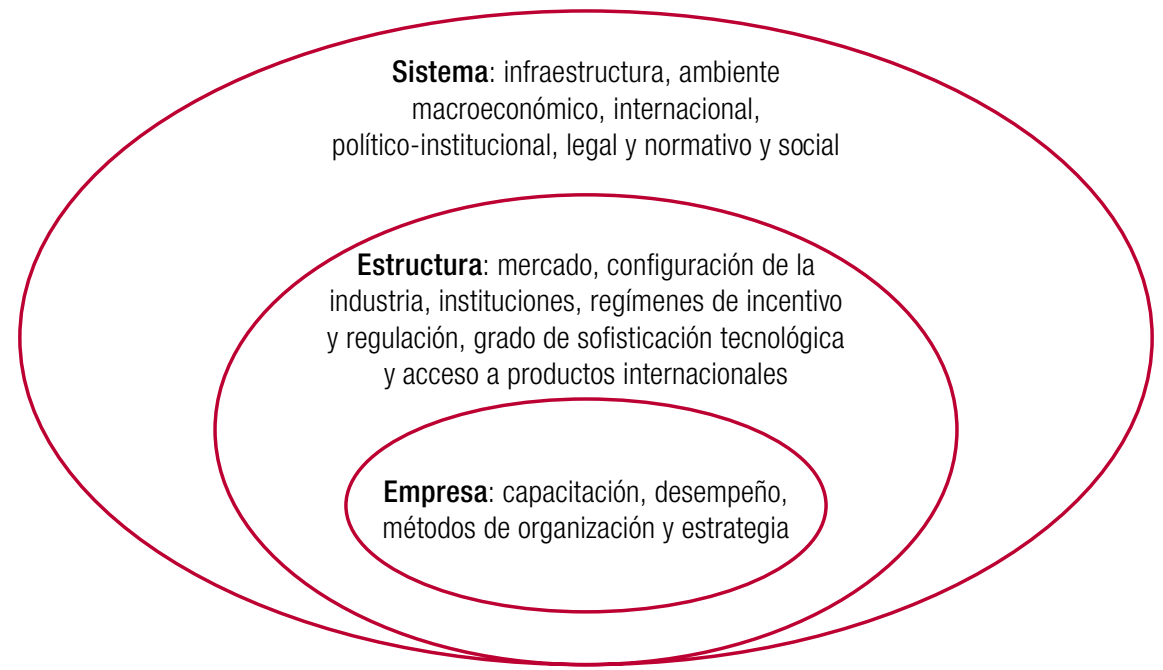

Fuente: Elaboración propia, sobre la base de J. Ferraz, D. Kupfer y L. Haguenauer, Made in Brazil: desafios competitivos para a indústria, Río de Janeiro, Campus, 1996.

En el contexto descrito en el diagrama 1, sería importante no solo definir las medidas de eficiencia (o competitividad) relativa entre los países, sino también los factores determinantes de su competitividad, con el fin de facilitar la formulación de políticas públicas para mejorar el ambiente competitivo de las empresas y ampliar sus consiguientes beneficios para la población en términos de crecimiento y desarrollo económico. 


\section{Metodología}

Para alcanzar los objetivos propuestos en este trabajo se utilizarán dos metodologías. Con la primera, el análisis envolvente de datos (DEA), se medirá el nivel de eficiencia de los factores de producción en los países en desarrollo. Además, como se trata de un panel de datos, se buscará evaluar la evolución de la productividad total de los factores de producción por medio del índice de Malmquist. A continuación, se utilizará un modelo Tobit para datos de panel que establecerá cuáles son los principales determinantes de la competitividad de los países de la muestra, con las medidas de eficiencia proporcionadas por la metodología DEA como variable dependiente. No obstante, como las variables explicativas del modelo Tobit tienen un alto grado de multicolinealidad, se construirán índices para captar su importancia mediante el análisis de componentes principales.

\section{Diferencias de eficiencia entre los países en desarrollo y sus determinantes: metodología}

\section{a) Medidas de eficiencia}

La metodología DEA genera medidas de eficiencia relativas entre las unidades de análisis, es decir, dentro de un determinado grupo muestral. Estas medidas se obtienen mediante el uso de programación lineal y cada unidad productiva se trata como una unidad de toma de decisiones (decision making unit-DMU).

En el análisis envolvente de datos, se asume que existen $m$ productos y $k$ insumos para cada una de las $n$ DMU. A partir de estos datos, se construyen las matrices de insumo (de dimensión $k \times n$ ) y de producto $(m \times n)$, que representarán las diversas unidades de toma de decisiones. Así, lo que propone esta metodología es obtener medidas de eficiencia a través de la razón entre los productos y los insumos, como sigue:

$$
\text { Eficiencia de la } D M U_{i}=\frac{u^{\prime} y_{i}}{v^{\prime} x_{i}}=\frac{u_{1} y_{1 i}+u_{2} y_{2 i}+\ldots+u_{m} y_{m i}}{v_{1} x_{1 i}+v_{2} x_{2 i}+\ldots+v_{k} x_{k i}}
$$

En que $u$ es un vector $(m \times 1)$ de pesos en los productos y $v$ es un vector $(k \times 1)$ de pesos en los insumos. Como los valores de las variables de producto e insumo pueden diferir sustancialmente entre las DMU, los pesos atribuidos para el alcance de las medidas de eficiencia también variarán. En consecuencia, es necesario establecer un problema que genere conjuntos adecuados de pesos para cada DMU, en comparación con las demás.

Como explican Ferreira y Gomes (2009), la formulación del problema debe tener en cuenta los valores de $u$ y $v$, de forma que se maximice la medida de eficiencia de cada DMU, sujeta a la restricción de que las medidas de todas las DMU sean iguales o inferiores a uno. Cuando se asumen rendimientos variables a escala ${ }^{5}$, se obtiene el siguiente problema de maximización:

sujeto a:

$$
\operatorname{Max}_{\theta, \lambda} \theta
$$

$$
\begin{aligned}
& -\theta y_{i}+Y \lambda \geq 0, \\
& x_{i}-X \lambda \geq 0 \\
& N_{1}^{\prime} \lambda=1 \\
& \lambda \geq 0
\end{aligned}
$$

5 Véanse más detalles en Charnes y otros (1994) y Cooper, Seiford y Tone (2000). 
$N_{1}^{\prime} \lambda=1$ es la restricción de convexidad y $N_{1}$ es un vector $(n \times 1)$ de guarismos unitarios. En que $1 / \theta$ es un escalar, cuyo valor será la medida de eficiencia de la i-ésima DMU. Si $\theta$ es igual a uno, la DMU se considera eficiente; en caso contrario, el valor será inferior a uno. El parámetro $\lambda$ es un vector $(n \times 1)$, cuyos valores se calculan para obtener la solución óptima.

Para una DMU eficiente, todos los valores de $\lambda$ serán cero y para una DMU ineficiente, los valores de $\lambda$ serán los pesos utilizados en la combinación lineal de otras DMU eficientes, que influyen en la proyección de la DMU ineficiente sobre la frontera calculada. Esto significa que, para una unidad ineficiente, existe por lo menos una unidad eficiente, cuyos pesos calculados proporcionarán la DMU virtual de la unidad ineficiente, mediante combinación lineal.

Por lo tanto, mediante la metodología DEA, se busca generar medidas de eficiencia de los países que representen su grado de competitividad en relación con los demás, pues tales medidas demostrarán el mayor nivel de producto posible, dada la asignación de los insumos (orientación al producto). De esta forma, se utilizarán las variables de insumo $X 1$ (número de personas empleadas) y $X 2$ (capital-paridad del poder adquisitivo) y la variable de producto $Y 1$ (producto interno bruto-paridad del poder adquisitivo).

\section{b) Índice de Malmquist y productividad total de los factores de producción}

El índice de Malmquist se utiliza para obtener estimaciones de la variación de la productividad total de los factores (PTF). Como se describe en el enfoque de Coelli, Rao y Battese (1998), el índice de PTF de Malmquist mide la variación de la PTF entre dos períodos, es decir, es una medida temporal de la productividad.

De acuerdo con el estudio pionero de Malmquist (1953), existen dos efectos que pueden afectar la productividad de los factores a lo largo del tiempo: i) el efecto de recuperación (catch-up), que determina la capacidad de una determinada DMU, dada una tecnología disponible, de aproximarse a la frontera de eficiencia; y ii) el efecto de desplazamiento, que capta el desplazamiento de la frontera de eficiencia temporalmente, es decir, un cambio en la tecnología que modifica la productividad. Por medio del índice de Malmquist, es posible calcular los efectos descritos de la siguiente forma:

$$
\text { Índice de Malmquist = efecto de recuperación } \times \text { efecto de desplazamiento }
$$

Además de obtener las medidas de eficiencia de los países en desarrollo, en el presente estudio se busca determinar si hubo evolución tecnológica o de la eficiencia técnica y, en consecuencia, verificar el comportamiento de la productividad total de los factores en el período 2011-2014. El índice de Malmquist complementa el análisis de eficiencia al indicar de manera más detallada las fuentes de la variación en la eficiencia de los países en desarrollo. En este caso, la aplicación del modelo incluye el año 2010, para poder obtener las variaciones de productividad a partir de 2011.

\section{Análisis econométrico}

\section{a) Análisis de componentes principales}

Dado el alto grado de multicolinealidad entre las variables explicativas utilizadas en el enfoque econométrico, muchas de ellas contienen la misma información. Para superar este problema, se optó por utilizar el análisis de componentes principales, una técnica que consiste en transformar un 
conjunto de variables originales en otro conjunto de variables de la misma dimensión denominadas componentes principales.

Los componentes principales se caracterizan por ser una combinación lineal de todas las variables originales, de manera que son independientes entre sí y se estiman con el fin de contener, en el orden en que se estiman, la máxima información contenida en los datos originales. Por lo tanto, el análisis de componentes principales se asocia con la idea de reducir la masa de datos con la menor pérdida posible de información.

Según Zivot y Wang (2003), al denominar $\hat{\Omega}$ la matriz de covarianza de las variables y $Z$ la matriz de las variables originales después de una normalización, el primer componente principal está dado por $x_{1}^{*} Z$ en que $x_{1}^{*}$ es la solución para:

$$
\max _{x_{1}} x_{1}^{\prime} \hat{\Omega} x_{1} \text { s.a. } x_{1}^{\prime} x_{1}=1
$$

Así, la solución $x_{1}^{*}$ es el valor asociado con el mayor autovalor de $\hat{\Omega}$. El segundo componente es $x_{2}^{*} Z$, y $x_{2}^{*}$ es el vector que resuelve la siguiente expresión:

$$
\max _{x_{2}} x_{2}^{\prime} \hat{\Omega} x_{2} \text { s.a. } x_{2}^{\prime} x_{2}=1 \text { e } x_{1}^{*^{\prime}} x_{2}=0
$$

De acuerdo con la solución anterior, se calculan los K componentes principales para $\mathrm{K}$ variables originales. De esta manera, se reducirá la dimensión de las variables dependientes del modelo censurado, Tobit, a partir del criterio de que se mantendrán los componentes que representan el $80 \%$ de la varianza de las variables originales del problema.

Por último, se recuperan los elementos de $x_{k}^{*}$ de forma de definir la importancia de cada una de las variables llamadas originales para la formación de los componentes y, en consecuencia, el impacto de cada variable en la competitividad. Este mecanismo se realizará mediante una estimación de mínimos cuadrados ordinarios simple de $x_{k}^{*}$ en $Z$.

\section{b) Modelo de regresión con datos censurados y remuestreo}

Para evaluar los determinantes de la competitividad de los países en desarrollo se estimará un modelo Tobit. La elección de este modelo se debe a que las medidas de eficiencia calculadas mediante el análisis envolvente de datos consisten en una muestra censurada, pues están limitadas en el intervalo entre 0 y 1 . En este caso, al proceder a una estimación por medio de mínimos cuadrados ordinarios, los parámetros serían inconsistentes y la inferencia estadística sobre ellos inviable (Wooldridge, 2011).

La censura puede suponer la pérdida o falta de información y la muestra puede presentar censura en el límite inferior (a la izquierda) o en el límite superior (a la derecha). Al describir $Y$ como la variable dependiente, $I$ como una constante que representa el valor mínimo asumido por $Y S$ el valor máximo de la variable $Y$, se advierte que $Y$ se observará solo en el intervalo $[I, S]$, de manera que las observaciones se consideran censuradas en los puntos inferior $(I)$ y superior $(S)$.

\footnotetext{
6 La inconsistencia de los parámetros deriva de la violación de los siguientes presupuestos del modelo de regresión lineal clásico: el término de error no sería independiente e idénticamente distribuido y no tendría una media igual a cero.
} 
A partir de las notaciones descritas en el párrafo anterior y sumando el vector $Z^{\prime}$ de variables explicativas, $\beta$ el vector de parámetros que se han de estimar, $Y^{\star}$ el vector estimado de $Y \varepsilon$ el término de error aleatorio, el modelo Tobit se puede describir de la siguiente manera:

$$
\begin{aligned}
& Y^{*}=Z^{\prime} \beta+\varepsilon_{i} \\
& Y=I, \text { si } Y^{*} \leq 1 \\
& Y=Y^{*}, \text { si } I<Y^{*}<S \\
& Y=S, \text { si } Y^{*} \geq S
\end{aligned}
$$

La estimación se realiza mediante el método de máxima verosimilitud. En este caso, hay dos etapas de estimación: en la primera se utiliza un modelo probit para el tratamiento de las observaciones censuradas y en la segunda se especifica un modelo lineal para las observaciones no censuradas. Los coeficientes estimados pueden interpretarse como el efecto de los regresores sobre la variable $Y^{\star}$.

Además, cabe destacar que se utilizará el modelo Tobit con datos de panel. Estos modelos, a su vez, permiten el examen de los efectos fijos o aleatorios de un determinado país o de los períodos de tiempo en las medidas de eficiencia (Park, 2005). Sin embargo, conforme Cameron y Trivedi (2005) y Da Silva y otros (2015), existen algunos problemas que dificultan el uso del modelo Tobit con efectos fijos, como la inconsistencia de los estimadores para paneles cortos, como en este estudio, y en los casos en que los efectos individuales no son uniformes. Como solución, los autores proponen el uso del modelo con efectos aleatorios.

En el caso del modelo de efectos aleatorios que se utilizará en este estudio, se asume que el efecto aleatorio es idéntico para todos los períodos y que no está correlacionado con los demás regresores, que los coeficientes angulares son los mismos para todos los años y grupos y que el componente estocástico del modelo no está correlacionado entre los períodos de tiempo (Greene, 2007).

No obstante, cabe señalar que el estimador Tobit solo será consistente en presencia de errores homocedásticos y normalmente distribuidos (Gujarati y Porter, 2011). En caso de heterocedasticidad o no normalidad de los errores, sería indicado emplear el método de bootstrap, que utiliza una determinada muestra varias veces para obtener las distribuciones muestrales de los parámetros de interés, obteniendo, en consecuencia, estimaciones robustas.

\section{Fuente y tratamiento de los datos}

Las variables $X 1, X 2$ e $Y 1$, utilizadas en la metodología DEA, se obtendrán a partir de la base de datos Penn World Table 9.07. Los países considerados en la muestra corresponden a la clasificación de países en desarrollo del Fondo Monetario Internacional $(\mathrm{FMI})^{8}$. Al excluir a los países para los cuales no se disponía de información, la muestra utilizada en la aplicación del modelo DEA quedó conformada por 82 países.

La medida de eficiencia generada en el análisis envolvente de datos se utilizará como variable sustitutiva de la competitividad nacional en la estimación del modelo Tobit. El vector de variables explicativas en el análisis econométrico describirá los factores determinantes de la competitividad, conforme el análisis de Ferraz, Kupfer y Haguenauer (1996), subdivididos en factores empresariales, estructurales y sistémicos. La síntesis de las variables utilizadas en el trabajo y las respectivas fuentes se describen en los cuadros A1.1, A1.2, A1.3, A1.4 y A1.5 del anexo A1. Se pueden ver más detalles al respecto en World Economic Forum (2014).

\footnotetext{
7 Disponible en https://www.rug.nl/ggdc/productivity/pwt/earlier-releases.

8 Véase http://www.imf.org/external/pubs/ft/weo/2014/01/pdf/text.pdf.
} 
Las variables Busin e Inov captan los factores empresariales y se refieren al ambiente de conexión entre las empresas y su capacidad de adoptar estrategias competitivas y a la capacidad de las empresas de innovar e implementar prácticas innovadoras, respectivamente. De esta forma abordan, en última instancia, la capacidad de las empresas de un país determinado de implementar estrategias competitivas.

Con respecto a los factores estructurales, las variables Inst, Demand, Financ, Techn y Msize incluyen, respectivamente, el papel de la calidad de las instituciones, las condiciones de la demanda, el nivel de desarrollo del mercado financiero y sus instituciones, la disponibilidad de tecnología avanzada y el tamaño del mercado en la competitividad nacional.

Por último, las variables Infra, Macro, Social, Train, Labor y Compet captan aspectos relativos a la oferta y la calidad de la infraestructura, el ambiente macroeconómico, la educación primaria y la salud, la capacitación de la mano de obra y la educación superior, la eficiencia del mercado de trabajo, la calidad del mercado financiero y la eficiencia en el mercado de bienes y servicios, respectivamente, y se relacionan con los factores sistémicos.

Tanto en la aplicación del análisis envolvente de datos como en el análisis econométrico, las variables se eligieron de acuerdo con la literatura especializada. Entre los autores que utilizaron variables similares se encuentran Ülengin y otros (2011), De Paula y Da Silva (2015), Rocha, Rebelatto y Camioto (2015), Charles y Zegarra (2014) y Lábaj, Luptáčik y Nežinský (2013).

\section{Resultados y discusión}

\section{Análisis envolvente de datos e índice de Malmquist}

A partir de la aplicación del modelo DEA, fue posible verificar el comportamiento de los países en desarrollo en el período 2011-2014, considerando una función de producción agregada con el capital y el trabajo como insumos y el producto interno bruto (PIB) como producto.

En el cuadro 1 se presentan los promedios de eficiencia, variación de la PTF y su respectiva descomposición en variación de la eficiencia técnica ( $\Delta$ Eficiencia), o efecto de recuperación, y variación tecnológica ( $\triangle$ Tecnología), o efecto de desplazamiento. La medida de eficiencia máxima (igual a 1) muestra que el país se encuentra en la frontera de la tecnología, es decir, que asigna sus recursos sin desperdicio, dada la tecnología más avanzada utilizada. El primer resultado que se muestra en el cuadro 1 indica que existe la posibilidad de que gran parte de los países en desarrollo asignen sus recursos de manera más eficiente y, en consecuencia, sean más competitivos.

\section{Cuadro 1}

Promedios de eficiencia, efecto de recuperación, efecto de desplazamiento y variación de la productividad total de los factores (PTF): muestra total, datos anuales y promedio para el período 2011-2014

\begin{tabular}{ccccc}
\hline Año/Medida & Eficiencia & $\Delta$ Eficiencia & $\Delta$ Tecnología & PTF \\
\hline 2011 & 0,571 & 0,858 & 1,193 & 1,023 \\
\hline 2012 & 0,558 & 0,968 & 1,039 & 1,006 \\
\hline 2013 & 0,578 & 1,068 & 0,918 & 0,980 \\
\hline 2014 & 0,597 & 1,081 & 0,903 & 0,977 \\
\hline Media & 0,572 & 0,996 & 1,013 & 0,999 \\
\hline
\end{tabular}

Fuente: Elaboración propia. 
Con respecto a la variación de la eficiencia técnica, se observa un cambio negativo en 2011 y 2012, tendencia que se invierte en los años siguientes, cuando el cambio se vuelve positivo en 2013 y 2014. Con la variación tecnológica ocurre lo contrario, pues la medida aumenta en 2011 y 2012 y disminuye en 2013 y 2014.

En general, el índice acumulado de productividad total de los factores indica que, en promedio, hubo una pequeña disminución $(0,99)$ de la productividad en los países en desarrollo. Este resultado parece indicar que, conforme Young (1994), si hubo crecimiento económico en los países analizados, este se explica por la expansión del uso de los insumos capital y trabajo y no por mejoras tecnológicas. Este resultado corrobora el estudio realizado por Marinho y Bittencourt (2007), que explican la escasa evolución de la productividad total de los factores de los países en desarrollo, y específicamente en América Latina, debido al poco énfasis de dichos países en las políticas relacionadas con la incorporación sistemática de innovaciones tecnológicas, el reconocimiento de las restricciones a la formación de ahorro interno, el alto grado de proteccionismo a las industrias nacientes y el carácter introvertido del proceso de desarrollo.

La disminución moderada de la PTF, a su vez, se explica principalmente por las variaciones negativas en la eficiencia técnica. Este resultado parece indicar que, en promedio, no hubo un proceso de convergencia en el período 2011-2014 entre los países analizados, es decir, los países rezagados en términos de producción eficiente no lograron alcanzar a los países más eficientes.

En el análisis regional se puede observar (véase el cuadro 2) que hubo diferencias sustanciales entre las regiones analizadas en el período 2011-2014. El nivel medio de eficiencia del 57,6\% indica que muchos países tienen margen para mejorar en términos de asignación de recursos. Los países asiáticos y europeos fueron los que presentaron, en promedio, los mejores resultados, con una eficiencia media del $68,5 \%$ y el $65,7 \%$, respectivamente. En contrapartida, los países africanos y latinoamericanos presentaron los peores indicadores en promedio (alrededor del 46,4\% y el $49,9 \%$, respectivamente).

Cuadro 2

Promedios de eficiencia, efecto de recuperación, efecto de desplazamiento y variación de la productividad total de los factores (PTF), por regiones, promedios para el período 2011-2014

\begin{tabular}{lcccc}
\hline Muestra & Eficiencia & $\Delta$ Eficiencia & $\Delta$ Tecnología & PTF \\
\hline Total & 0,576 & 0,989 & 1,007 & 0,996 \\
\hline Europa & 0,657 & 1,006 & 1,016 & 1,010 \\
\hline América Latina y el Caribe & 0,499 & 0,986 & 1,017 & 0,991 \\
\hline Asia & 0,685 & 0,996 & 1,014 & 1,001 \\
\hline África & 0,464 & 1,001 & 1,008 & 0,999 \\
\hline
\end{tabular}

Fuente: Elaboración propia.

Con respecto a la productividad total de los factores de producción, se observa que solo los países europeos registraron un promedio de variación positiva de la PTF en el período analizado, mientras en el caso de los países asiáticos y africanos este se mantuvo prácticamente constante. Por otra parte, el promedio de los países latinoamericanos empeoró, lo que indica un rezago relativo en relación con los demás países de la muestra en el período analizado.

El desempeño inferior de los países africanos y latinoamericanos en términos de eficiencia y variación de la eficiencia puede explicarse en parte por los peores indicadores de factores empresariales, estructurales y sistémicos en relación con los países europeos y asiáticos. Con respecto a los factores empresariales (sofisticación del mercado y grado de innovación), del gráfico 1 surge que los países africanos presentaron los valores más bajos para los indicadores Busin e Inov. Los países de América Latina también registraron el peor indicador de innovación (Inov), idéntico al de los países africanos. 


\section{Gráfico 1}

Factores empresariales: promedio de los valores para el período 2011-2014

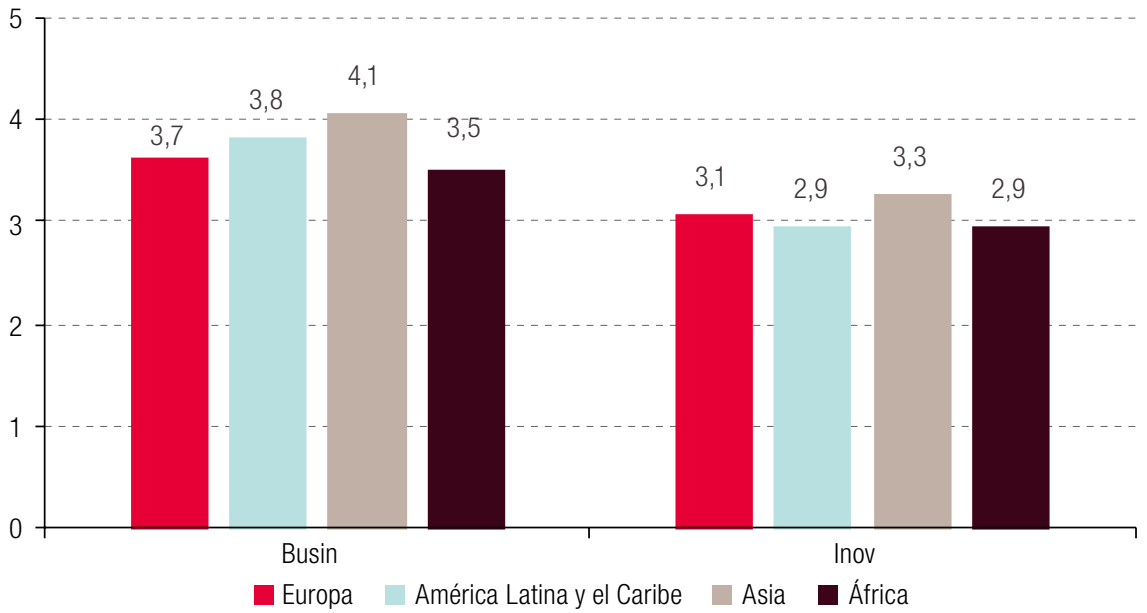

Fuente: Elaboración propia, sobre la base de Foro Económico Mundial, “The Global Competitiveness Index”, 2014.

Con respecto a los factores estructurales, se observa una vez más (véase el gráfico 2) que los países de Asia presentan los mejores indicadores, en contraste con los países africanos y latinoamericanos, que registran los peores indicadores en cuanto a la calidad de las instituciones, las condiciones de la demanda, la calidad del sistema financiero, la disponibilidad de tecnología avanzada y el tamaño del mercado. Resultados similares se observan con respecto a los factores sistémicos (véase el gráfico 3), que indican que, en general, los países que alcanzaron los mejores promedios de eficiencia en el enfoque DEA son los mismos que presentan los mejores indicadores de infraestructura, ambiente macroeconómico, educación básica y salud, capacitación técnica y enseñanza superior, nivel de competencia y legislación laboral.

\section{Gráfico 2}

Factores estructurales: promedio de los valores para el período 2011-2014

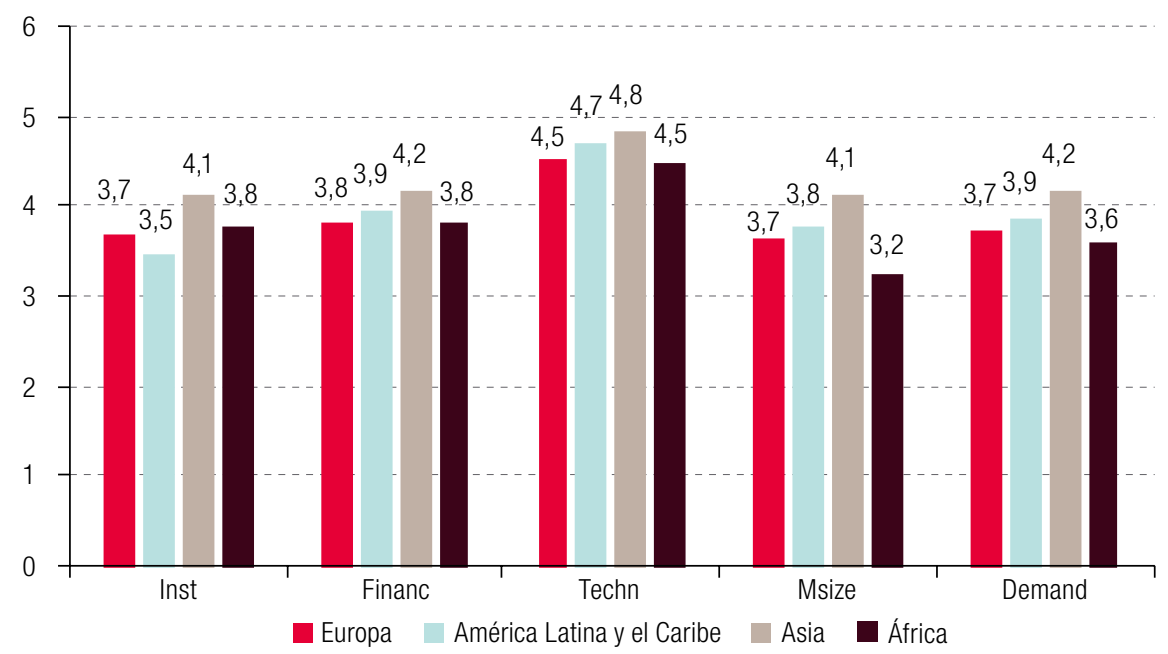

Fuente: Elaboración propia, sobre la base de Foro Económico Mundial, “The Global Competitiveness Index”, 2014. 


\section{Gráfico 3}

Factores sistémicos: promedio de los valores para el período 2011-2014

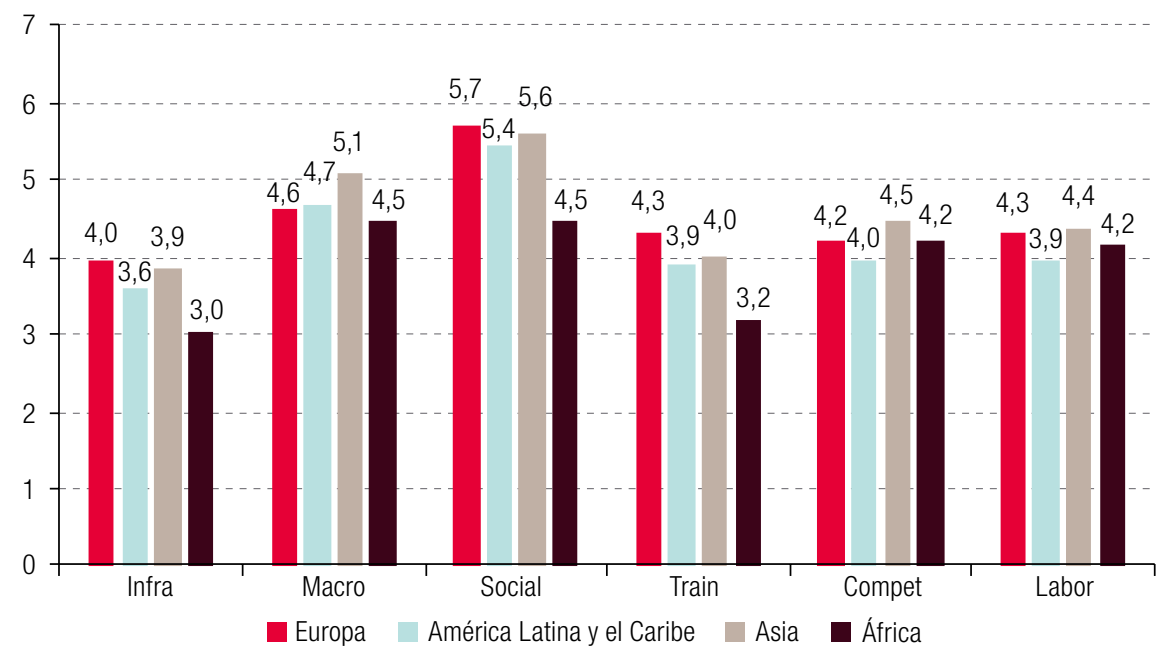

Fuente: Elaboración propia, sobre la base de Foro Económico Mundial, "The Global Competitiveness Index", 2014.

Por último, los países con el mejor desempeño en términos de eficiencia técnica fueron: Arabia Saudita, Azerbaiyán, Brunei Darussalam, China, Federación de Rusia, Kuwait, Montenegro y Qatar, que tuvieron una medida de eficiencia igual a 1 en todos los años analizados. Estos países se caracterizan porque todos los indicadores empresariales, estructurales y sistémicos promedio son mayores con respecto al promedio de la muestra total, como se puede observar en el cuadro 3.

\section{Cuadro 3}

Promedio de los indicadores empresariales, estructurales y sistémicos: muestra total y países con mejores puntajes de eficiencia, 2011-2014

\begin{tabular}{|c|c|c|c|c|c|c|c|c|}
\hline \multicolumn{3}{|c|}{ Factores empresariales } & \multicolumn{3}{|c|}{ Factores estructurales } & \multicolumn{3}{|c|}{ Factores sistémicos } \\
\hline Indicador & Total & Mejores & Indicador & Total & Mejores & Indicador & Total & Mejores \\
\hline Busin & 3,79 & 4,18 & Inst & 3,77 & 4,49 & Infra & 3,60 & 4,49 \\
\hline \multirow[t]{5}{*}{ Inov } & 3,06 & 3,56 & Financ & 3,96 & 4,28 & Macro & 4,74 & 5,98 \\
\hline & & & Techn & 4,65 & 4,86 & Social & 5,30 & 5,87 \\
\hline & & & Msize & 3,72 & 4,16 & Train & 3,84 & 4,41 \\
\hline & & & Demand & 3,87 & 4,28 & Compet & 4,23 & 4,48 \\
\hline & & & & & & Labor & 4,20 & 4,65 \\
\hline
\end{tabular}

Fuente: Elaboración propia, sobre la base de Foro Económico Mundial, "The Global Competitiveness Index", 2014.

Al igual que los análisis realizados por Ferraz, Kupfer y Haguenauer (1996), IDM (2012) y World Economic Forum (2014), los resultados descritos indican que la mejora de los indicadores empresariales, estructurales y sistémicos puede redundar en altos niveles de competitividad y eficiencia en los países en desarrollo.

En síntesis, los resultados obtenidos mediante el análisis envolvente de datos indican que hay margen para que la gran mayoría de los países en desarrollo analizados mejoren en términos de asignación eficiente de sus recursos, en particular los países africanos y latinoamericanos. Por último, se observó que los países que alcanzaron promedios de eficiencia más altos también tienen mejores indicadores empresariales, estructurales y sistémicos. 
Sin embargo, cabe destacar que algunos factores ambientales o variables contextuales pueden influir de forma significativa en los puntajes de eficiencia. Dado que la competitividad depende de factores estructurales, empresariales y sistémicos, esta diversidad de factores merece evaluarse en su relación con la mejora de las medidas de eficiencia. Así, en el apartado siguiente se busca estimar, mediante un modelo de datos censurados, el impacto de las diversas variables en la eficiencia de los países en desarrollo.

\section{Análisis econométrico}

El primer procedimiento del análisis estadístico consistió en reducir la masa de datos mediante el análisis de componentes principales, dado el umbral que se impuso para mantener en el análisis solo los componentes que respetaran el criterio de Kaiser, es decir, que tuvieran autovalores superiores a uno ${ }^{9}$. Por lo tanto, como se muestra en el cuadro 4 , se mantuvieron 3 de los 13 componentes principales.

Cuadro 4

Análisis de componentes principales

\begin{tabular}{lccc}
\hline Componente & Proporción & Acumulativo & Autovalor \\
\hline Componente 1 (PC1) & 0,5730 & 0,5730 & 7,4491 \\
\hline Componente 2 (PC2) & 0,1270 & 0,7000 & 1,6512 \\
\hline Componente 3 (PC3) & 0,0793 & 0,7793 & 1,0308 \\
\hline Componente 4 (PC4) & 0,0630 & 0,8423 & 0,8192 \\
\hline Número de observaciones: 321 & & & \\
\hline
\end{tabular}

Fuente: Elaboración propia.

A partir del análisis de componentes principales, se estimó un modelo de datos censurados, Tobit, con las medidas de eficiencia generadas por la metodología DEA como variable dependiente y los componentes como variables explicativas. Cabe destacar que el modelo de datos censurados se estimó con el método de bootstrap, que está indicado para resolver o minimizar los problemas derivados de la dificultad para determinar un patrón asintótico de los errores, que tiende a subestimar la verdadera varianza. De esta forma, el modelo se estimó por medio del método de bootstrap, utilizando estimaciones Tobit con efectos aleatorios y agrupados (pooled).

Los resultados de las estimaciones se presentan en el cuadro 5. Se observa que no hubo diferencias sustanciales (solo en la magnitud de los errores estándar) entre las estimaciones agrupadas y con efectos aleatorios y los resultados fueron idénticos en términos de significación y signo de las variables.

Del cuadro 5 surge que los dos primeros componentes resultaron significativos en el sentido de influir en la competitividad de un determinado país en desarrollo, ambos con efectos positivos. Así, el objetivo de este trabajo, a partir de este punto, fue recuperar los pesos utilizados para la formación de los componentes con el fin de extraer información sobre la influencia de las variables originales.

9 Véase más información sobre los criterios de selección de los componentes en Fávero y otros (2009). 


\section{Cuadro 5}

Determinantes de la competitividad de los países en desarrollo: estimación Tobit y mínimos cuadrados ordinarios, 2013

\begin{tabular}{lcc}
\hline Variable/Modelo & Efectos aleatorios & $\begin{array}{c}\text { Agrupados } \\
\text { (pooled) }\end{array}$ \\
\hline PC1 & 0,0240 & 0,0240 \\
\hline PC2 & $(0,0048)^{\star \star \star}$ & $(0,0042)^{\star \star \star}$ \\
\hline PC3 & 0,0349 & 0,0349 \\
\hline Constante & $(0,0109)^{\star \star \star}$ & $(0,0111)^{\star \star \star}$ \\
\hline Observaciones & $-0,0153$ & $-0,0153$ \\
\hline Número de observaciones censuradas a la izquierda & $(0,0164)$ & $(0,0172)$ \\
\hline Número de observaciones censuradas a la derecha & 0,6029 & 0,6029 \\
\hline Wald chi2 & $(0,0304)^{\star \star \star}$ & $(0,0321)^{\star \star \star}$ \\
\hline Prob>chi2 & 321 & 321 \\
\hline Futen & 0 & 41 \\
\hline
\end{tabular}

Fuente: Elaboración propia.

Nota: $\left.\quad{ }^{(\star \star}\right)$ significativo al 1\%; errores estándar bootstraping entre paréntesis.

Para recuperar el peso de cada variable en la formación de los componentes principales, estas se normalizaron y luego se estimó un modelo por medio de mínimos cuadrados ordinarios, con los componentes como variables dependientes. Dado que los componentes no son más que combinaciones lineales de las variables originales del modelo, $R^{2}$ de hecho debe ser $1 \mathrm{y}$, en consecuencia, no hay error estándar para los coeficientes.

Después de aplicar el procedimiento descrito en el párrafo anterior, para el primer componente (PC1) - que generó un efecto positivo en la competitividad de los países en desarrollo (véase el cuadro 6) - , todas las variables tuvieron un impacto positivo en la competitividad. Los mayores pesos se dan a variables como el ambiente de negocios y la capacidad innovadora de las empresas, y a aspectos como la infraestructura, las condiciones y la calidad de la demanda, el ambiente competitivo, la salud, la educación básica y el nivel de capacitación de la mano de obra, las instituciones y el sistema financiero y el acceso a tecnologías avanzadas.

Cuadro 6

Peso de cada variable principal en la formación de los componentes principales

\begin{tabular}{|c|c|c|c|}
\hline Variable original/componente & Tipo de factor & PC1 & PC2 \\
\hline Busin & \multirow[t]{2}{*}{ Empresarial } & 0,3382 & 0,0449 \\
\hline Inov & & 0,3163 & 0,0338 \\
\hline Inst & \multirow[t]{5}{*}{ Estructural } & 0,3079 & $-0,2498$ \\
\hline Financ & & 0,2991 & $-0,1840$ \\
\hline Techn & & 0,3133 & $-0,1225$ \\
\hline Demand & & 0,3256 & 0,0289 \\
\hline MSize & & 0,1207 & 0,5248 \\
\hline Infra & \multirow[t]{7}{*}{ Sistémico } & 0,3148 & 0,1688 \\
\hline Macro & & 0,1986 & 0,1040 \\
\hline Compet & & 0,2838 & $-0,3664$ \\
\hline Social & & 0,2164 & 0,3991 \\
\hline Train & & 0,2797 & 0,3366 \\
\hline Labor & & 0,1950 & $-0,4035$ \\
\hline Observaciones & & 321 & 321 \\
\hline
\end{tabular}

Fuente: Elaboración propia. 
El segundo componente también tiene un efecto positivo en la competitividad. Las variables con mayor peso en este componente son: MSize, Social, Train, Compet y Labor. El impacto de las tres primeras en la competitividad es positivo y el de las dos últimas negativo.

Para facilitar el análisis del cuadro 6, los casilleros señalados en gris oscuro indican las variables con un peso importante en la determinación del componente, mientras los casilleros señalados en gris claro indican una menor importancia de la variable en la determinación del componente. Se analizaron aquellas variables que obtuvieron signos equivalentes en relación con la variable dependiente competitividad y que fueron importantes en la determinación de los componentes (es decir, las variables Busin, Inov, Demand, MSize, Infra, Macro, Social y Train). En cierta forma, el resto de las variables presenta un comportamiento ambiguo, al tener coeficientes contrarios en los componentes 1 y 2 (PC1 y PC2). Con esta perspectiva, no se realizará un análisis más profundo de sus posibles efectos en la eficiencia. Este es el caso de las variables Inst, Financ, Techn, Compet y Labor.

Los resultados obtenidos pueden interpretarse conforme tres vertientes. En primer lugar, se observa la importancia de los factores empresariales, caracterizada por el peso sustancial de las variables Busin e Inov en el PC1. En consecuencia, se comprueba el beneficio para la competitividad que deriva de un ambiente de negocios sofisticado - es decir, caracterizado por el gran número y la calidad de los proveedores locales, la amplitud de la cadena de valor, el control de la distribución internacional, la sofisticación del proceso de producción, la extensión de la comercialización, entre otros - y de la capacidad innovadora de las empresas, es decir, de la calidad de las instituciones de investigación científica, el gasto de las empresas en l+D, la colaboración entre universidades e industrias en I+D, las compras gubernamentales de productos de tecnología avanzada, la disponibilidad de científicos e ingenieros, las solicitudes de patentes y la protección de la propiedad intelectual, entre otros. Estos resultados corroboran el análisis teórico realizado, por ejemplo, por Romer (1990) y Aghion y Howitt (1992).

En segundo lugar, con respecto a los factores estructurales, se destacan aspectos como el tamaño del mercado (MSize) y, en menor magnitud, la calidad de la demanda (Demand), que se observa en el grado de orientación al cliente y la sofisticación del comprador. Este resultado corrobora el análisis teórico realizado por Romer (1986) y Ferraz, Kupfer y Haguenauer (1996), entre otros.

Además, la variable MSize tuvo un peso considerable en el PC2, que indica que las economías de escala generadas por los grandes mercados nacionales y de exportación son factores importantes para que los países logren mejores resultados en términos de competitividad. Este resultado evidencia los análisis teóricos realizados por autores como Grosmman y Helpman (1991), que indican la importancia de la apertura comercial para el proceso de crecimiento a través de una mayor competitividad.

En tercer lugar, con respecto a los factores sistémicos, las variables relevantes para la explicación de los componentes principales fueron Infra, Social, Train y, en menor magnitud, Macro. Así, se señala el papel fundamental de la mejora de los medios de transporte, la calidad de la oferta de energía y los servicios de telecomunicaciones, además de la provisión de salud, educación a nivel básico y superior y capacitación de la mano de obra. Estos resultados corroboran los análisis teóricos realizados, por ejemplo, por Lucas (1988) y Barro (1990), que hacen hincapié en la contribución de la infraestructura y el capital humano al desarrollo de los países. Asimismo, los aspectos relacionados con el saldo presupuestario del gobierno, el ahorro nacional bruto, el control de la inflación y de la deuda pública, además de la calificación crediticia del país, serían importantes para que los países en desarrollo fueran competitivos.

En síntesis, el análisis econométrico evidenció la importancia del capital humano, la infraestructura, las economías de escala, la estabilidad macroeconómica y la capacidad innovadora de las empresas para lograr un ambiente más competitivo en los países en desarrollo. Estos resultados 
corroboran el análisis teórico de Ferraz, Kupfer y Haguenauer (1996) y coinciden con los argumentos de los teóricos del crecimiento endógeno y otros. En relación con otra evidencia empírica, aunque indirectamente, los resultados son similares a los obtenidos por Rocha, Rebelatto y Camioto (2015), De Paula y Da Silva (2015) y Charles y Zegarra (2014), al indicar que los factores ambientales son importantes para que los países sean competitivos.

\section{Conclusiones}

El objetivo del presente trabajo era determinar el nivel de competitividad y sus principales determinantes en los países en desarrollo, en un enfoque que analiza la competitividad en un concepto ex ante, es decir, se considera competitivo al país que utiliza sus insumos capital y trabajo de manera de generar el mayor producto posible, en relación con los demás países.

Sobre la base de la metodología DEA, se obtuvo el nivel de eficiencia técnica y de escala de los países en desarrollo. Los resultados mostraron que gran parte de los países de la muestra tiene margen para mejorar en términos de asignación eficiente de recursos, visto el bajo nivel medio de eficiencia. También se observó que los países y regiones que alcanzaron mayores promedios de eficiencia y evolucionaron en términos de productividad total de los factores, según el índice de Malmquist, presentan mejores indicadores empresariales, estructurales y sistémicos.

Con respecto al análisis econométrico, el modelo Tobit indicó que factores empresariales como la capacidad innovadora y la sofisticación del ambiente de negocios, aspectos estructurales como el tamaño de los mercados interno y externo y la calidad de la demanda, además de factores sistémicos como la oferta y la calidad de la infraestructura, la salud, la educación básica y superior, la capacitación de la mano de obra y el ambiente macroeconómico son importantes para que los países en desarrollo sean más competitivos. Estas afirmaciones se basan en el análisis de componentes principales, pues esas variables tuvieron un peso relevante en la determinación de los componentes.

Se destaca la importancia de las autoridades políticas y económicas en esos países, pues la prestación de servicios de infraestructura económica, salud y educación y el mantenimiento de un ambiente macroeconómico favorable pueden contribuir a que los países alcancen altos niveles de competitividad y, en consecuencia, disfruten de sus beneficios en términos de desarrollo económico y humano. De esta forma, se espera que la estructura en la que se insertan las empresas sea más adecuada para su funcionamiento, por medio de una mayor calidad de los aspectos de la demanda y del tamaño del mercado. En última instancia, se espera que estos factores promuevan un ambiente empresarial adecuado para la toma de decisiones de la empresa, que podrá actuar en un ambiente de negocios sofisticado y propicio para la innovación, generando mayor competitividad para el país.

\section{Bibliografía}

Aghion, P. y P. Howitt (1992), "A model of growth through creative destruction", Econometrica, vol. 60, № 2, Hoboken, Wiley.

Araujo, J., D. Feitosa y A. da Silva (2014), "América Latina: productividad total de los factores y su descomposición”, Revista CEPAL, Nº 114 (LC/G.2629-P), Santiago, Comisión Económica para América Latina y el Caribe (CEPAL).

Barro, R. (1990), "Government spending in a simple model of economic growth", The Journal of Political Economy, vol. 98, № 5, Chicago, The University of Chicago Press.

Cameron, A. y P. Trivedi (2005), Microeconometrics: Methods and Applications, Cambridge, Cambridge University Press. 
Charles, V. y L. Zegarra (2014), "Measuring regional competitiviness through data envelopment analysis: a Peruvian case", Expert Systems with Applications, vol. 41, № 11, Amsterdam, Elsevier.

Charnes, A. y otros (eds.) (1994), Data Envelopment Analysis: Theory, Methodology, and Application, Dordrecht, Kluwer Academic Publishers.

Coelli, T., D. Rao y G. Battese (1998), An Introduction to Efficiency and Productivity Analysis, Dordrecht, Kluwer Academic Publishers.

Cooper, W., L. Seiford y K. Tone (2000), Data Envelopment Analysis: A Comprehensive Text with Models, Applications, References and DEA-Solver Software, Boston, Massachusetts, Kluwer Academic Publishers.

Da Silva, M. y others (2015), "Determinants of out-of-pocket health expenditure on children: an analysis of the 2004 Pelotas Birth Cohort", International Journal for Equity in Health, vol. 14, № 53, Londres, BioMed Central.

De Paula, J. y O. da Silva (2015), "Fatores internos como determinantes da competitividade no comércio internacional: um enfoque gravitacional”, Revista Análise Econômica, vol. 33, № 64, Porto Alegre, Universidad Federal de Rio Grande do Sul.

Esser, K. y otros (1996), "Competitividad sistémica: nuevo desafío para las empresas y la política", Revista CEPAL, № 59 (LC/G.1931-P), Santiago, Comisión Económica para América Latina y el Caribe (CEPAL).

Fávero, L. y otros (2009), Análise de dados: modelagem multivariada para tomada de decisões, Río de Janeiro, Elsevier.

Feenstra, R., R. Inklaar y M. Timmer (2015), "The next generation of the Penn World Table", American Economic Review, vol. 105, № 10, Nashville, Tennessee, American Economic Association.

Ferraz, J., D. Kupfer y L. Haguenauer (1996), Made in Brazil: desafios competitivos para a indústria, Río de Janeiro, Campus.

Ferreira, C. y A. Gomes (2009), Introdução à análise envoltória de dados: teoria, modelos e aplicações, Viçosa, Editora UFV.

Foro Económico Mundial (2014), The Global Competitiveness Report 2013-2014, Ginebra.

Godfrey, N. (2008), "Why is Competition Important for Growth and Poverty Reduction?", ponencia presentada en el Foro Global de la OCDE sobre Inversión Internacional VII, Organización de Cooperación y Desarrollo Económicos (OCDE), marzo.

Greene, W. (2007), LIMDEP version 9.0: econometric modeling guide, Nueva York, Econometric Software.

Grossman, G. y E. Helpman (1991), "Quality ladders and product cycles", Quarterly Journal of Economics, vol. 106, N ${ }^{\circ}$ 2, Oxford, Oxford University Press.

Gujarati, D. y D. Porter (2011), Econometria básica, São Paulo, McGraw Hill.

Haguenauer, L. (1989), "Competitividade: conceitos e medidas: uma resenha da bibliografia recente com ênfase no caso brasileiro", Texto para Discussão, № 211, Río de Janeiro, Universidad Federal de Río de Janeiro.

IMD (Instituto para el Desarrollo Gerencial) (2012), World Competitiveness Yearbook 2012, Lausana.

Kordalska, A. y M. Olczyk (2016), "Global competitiveness and economic growth: a one-way or two-way relationship? Equilibrium”, Equilibrium. Quarterly Journal of Economics and Economic Policy, vol. 11, $N^{\circ} 1$, Varsovia, Instituto de Investigaciones Económicas (IBG).

Lábaj, M., M. Luptáčik y E. Nežinský (2013), "Data envelopment analysis for measuring of economic growth in terms of welfare beyond GDP", Department of Economic Policy Working Paper, No 2, Bratislava, Universidad de Economía de Bratislava.

Lucas, R. (1988), "On the mechanics of economic development", Journal of Monetary Economics, vol. 22, $N^{\circ} 1$, Amsterdam, Elsevier.

Malmquist, S. (1953), "Index numbers and indifference surfaces", Trabajos de Estadística y de Investigación Operativa, vol. 4, № 2, Nueva York, Springer.

Marinho, E. y A. Bittencourt (2007), "Produtividade e crescimento econômico na América Latina: a abordagem da fronteira de produção estocástica", Estudos Econômicos, vol. 37, № 1, São Paulo, Universidad de São Paulo.

Moura, R. (2009), "Impacto do IDE no crescimento econômico do país receptor - teoria e evidência empírica", tesis para optar al grado de magister, Universidad de Porto [en línea] https://www.fep.up.pt/cursos/ mestrados/megi/Tese_Rui\%20Moura_final.pdf.

OCDE (Organización de Cooperación y Desarrollo Económicos) (2002), Foreign Direct Investment for Development: Maximising Benefits, Minimising Costs, París.

Park, H. (2005), "Linear regression models for panel data using SAS, Stata, LIMDEP, and SPSS" [en línea] http://rt.uits.iu.edu. 
Ram, R. y K. Zhang (2002), "Foreign direct investment and economic growth: evidence form cross-country data for the 1990s", Economic Development and Cultural Change, vol. 51, № 1, Chicago, The University of Chicago Press.

Rocha, R., D. Rebelatto y F. Camioto (2015), "Análise da eficiência de fatores nos países do BRICS a partir da aplicação da Análise por Envoltória de dados", RACEF - Revista de Administração, Contabilidade e Economia da Fundace, vol. 6, № 1, São Paulo, Fundación de Investigación y Desarrollo de Administración, Contabilidad y Economía (FUNDACE).

Romer, P. (1990), "Endogenous technological change", The Journal of Political Economy, vol. 98, № 5, Chicago, The University of Chicago Press. (1986), "Increasing returns and long-run growth", The Journal of Political Economy, vol. 94, № 5, Chicago, The University of Chicago Press.

Ülengin, F. y otros (2011), "The competitiveness of nations and implications for human development", Socio-Economic Planning Sciences, vol. 45, № 1, Amsterdam, Elsevier.

Unión Europea (2001), Second Report on Economic and Social Cohesion, Bruselas.

Vissak, T. y T. Roolaht (2005), "The negative impact of foreign direct investment on the Estonian economy", Problems of Economic Transition, vol. 48, № 2, Abingdon, Taylor \& Francis.

Wooldridge, J. (2011), Introdução à econometria: uma abordagem moderna, São Paulo, Cengage Learning.

Young, A. (1994), "The tyranny of numbers: confronting the statistical realities of the east Asian growth experience", NBER Working Paper, № 4680, Cambridge, Massachusetts, Oficina Nacional de Investigaciones Económicas (NBER).

Zhang, K. (2001), "How does foreign direct investment affect economic growth in China?", Economics of Transition and Institutional Change, vol. 9, № 3, Hoboken, Wiley.

Zivot, E. y J. Wang (2003), Modeling Financial Time Series with S-Plus, Nueva York, Springer. 


\section{Anexo A1}

\section{Cuadro A1.1}

Variables utilizadas en el modelo de análisis envolvente de datos (DEA) y el índice de Malmquist

\begin{tabular}{ll}
\hline Variable en la fuente & Fuente \\
\hline $\begin{array}{ll}\text { Producto interno bruto (PIB) real basado en la producción a tasas } \\
\text { de paridad del poder adquisitivo encadenadas } \\
\text { (en millones de dólares de 2011) }\end{array}$ & $\begin{array}{l}\text { Penn World Table 9.0 } \\
\text { (Feenstra, Inklaar y Timmer (2015)) }\end{array}$ \\
\cline { 1 - 2 } Número de personas empleadas (en millones) & \\
\cline { 1 - 2 } Niveles de capital a paridad del poder adquisitivo actual (en millones de dólares de 2011) &
\end{tabular}

Fuente: Elaboración propia.

Cuadro A1.2

Variables utilizadas en el análisis de componentes principales y Tobit

\begin{tabular}{|c|c|c|c|}
\hline Índice & Nombre & Variable & Variable en la fuente \\
\hline \multirow[t]{2}{*}{ Empresarial } & Busin & $\begin{array}{l}\text { Índice de calidad de la sofisticación } \\
\text { de los negocios (1-7) }\end{array}$ & Pilar 11: Sofisticación de los negocios \\
\hline & Inov & Índice de calidad de las innovaciones (1-7) & Pilar 12: Innovación \\
\hline \multirow[t]{5}{*}{ Estructural } & Inst & Índice de calidad institucional (1-7) & Pilar 1: Instituciones \\
\hline & Financ & $\begin{array}{l}\text { Índice de calidad de desarrollo del } \\
\text { mercado financiero (1-7) }\end{array}$ & Pilar 8: Desarrollo del mercado financiero \\
\hline & Techn & Adopción de tecnología (1-7) & A. Adopción de tecnología \\
\hline & Msize & Índice de calidad del tamaño del mercado (1-7) & Pilar 10: Tamaño del mercado \\
\hline & Demand & Calidad de las condiciones de la demanda (1-7) & B. Calidad de las condiciones de la demanda \\
\hline \multirow[t]{6}{*}{ Sistémico } & Infra & Índice de calidad de la infraestructura (1-7) & Pilar 2: Infraestructura \\
\hline & Macro & Índice de calidad del ambiente macroeconómico (1-7) & Pilar 3: Ambiente macroeconómico \\
\hline & Social & Índice de calidad de la salud y la educación (1-7) & Pilar 4: Salud y educación primaria \\
\hline & Train & $\begin{array}{l}\text { Índice de calidad de la capacitación y } \\
\text { la escolarización avanzada (1-7) }\end{array}$ & Pilar 5: Educación superior y capacitación \\
\hline & Labor & $\begin{array}{l}\text { Índice de calidad de la eficiencia } \\
\text { del mercado de trabajo (1-7) }\end{array}$ & Pilar 7: Eficiencia del mercado de trabajo \\
\hline & Compet & Nivel y calidad de la competencia del mercado & A. Competencia \\
\hline
\end{tabular}

Fuente: Elaboración propia, sobre la base de Foro Económico Mundial, The Global Competitiveness Report 2013-2014, Ginebra, 2014.

\section{Cuadro A1.3}

Variables relacionadas con las variables utilizadas en el análisis de componentes principales: factores empresariales

\begin{tabular}{ll}
\hline Variable & Variables relacionadas \\
\hline $\begin{array}{l}\text { Busin: sofisticación del } \\
\text { ambiente de negocios }\end{array}$ & $\begin{array}{l}\text { Cantidad de proveedores locales, calidad de los proveedores locales, estado de desarrollo de } \\
\text { conglomerados, naturaleza de la ventaja competitiva, amplitud de la cadena de valor, control } \\
\text { de la distribución internacional, sofisticación del proceso de producción, extensión de la } \\
\text { comercialización, disposición para delegar autoridad, dependencia en la gestión profesional. }\end{array}$ \\
\hline Inov: capacidad innovadora & $\begin{array}{l}\text { Capacidad de innovación, calidad de las instituciones de investigación científica, gasto de las } \\
\text { empresas en investigación y desarrollo (l+D), colaboración entre universidades e industrias } \\
\text { en l+D, compras gubernamentales de productos de tecnología avanzada, disponibilidad de } \\
\text { científicos e ingenieros, solicitudes de patentes, protección de la propiedad intelectual. }\end{array}$ \\
\hline
\end{tabular}

Fuente: Elaboración propia, sobre la base de Foro Económico Mundial, The Global Competitiveness Report 2013-2014, Ginebra, 2014. 


\section{Cuadro A1.4}

Variables relacionadas con las variables utilizadas en el análisis de componentes principales: factores estructurales

\begin{tabular}{lll}
\hline & Instituciones públicas & Instituciones privadas \\
\hline Inst: aspectos & Derechos de propiedad, protección de la propiedad intelectual, & Comportamiento ético de las \\
institucionales & malversación de fondos públicos, confianza pública en los políticos, & empresas, solidez de las normas \\
& pagos irregulares y sobornos, independencia judicial, favoritismo en & de auditoría y elaboración de \\
& las decisiones de los funcionarios del gobierno, derroche del gasto & informes, eficacia de los consejos \\
público, regulación del gobierno, eficiencia del marco legal en la & de administración, protección de \\
& resolución de disputas, eficiencia del marco legal en reglamentos, & los intereses de los accionistas \\
& transparencia en la formulación de políticas gubernamentales, costos & minoritarios, solidez de la \\
& comerciales del terrorismo, costos comerciales de la criminalidad y la & protección de los inversionistas. \\
& violencia, crimen organizado, confiabilidad de los servicios policiales. &
\end{tabular}

\begin{tabular}{ll}
\hline $\begin{array}{l}\text { Demand: aspectos } \\
\text { cualitativos de la demanda }\end{array}$ & Grado de orientación al cliente, sofisticación del comprador. \\
\hline $\begin{array}{l}\text { Financ: aspectos } \\
\text { institucionales y de } \\
\text { acceso al crédito }\end{array}$ & Eficiencia del mercado financiero. \\
\cline { 2 - 2 } & $\begin{array}{l}\text { Disponibilidad de servicios financieros, financiamiento a } \\
\text { través del mercado de valores local, facilidad de acceso } \\
\text { a préstamos, disponibilidad de capital de riesgo. }\end{array}$ \\
\hline $\begin{array}{l}\text { Techn: disponibilidad } \\
\text { de tecnología }\end{array}$ & $\begin{array}{l}\text { Adopción de tecnología. } \\
\text { Disponibilidad de tecnologías recientes, absorción de tecnología a nivel de } \\
\text { la empresa, inversión extranjera directa (IED) y transferencia de tecnología. }\end{array}$
\end{tabular}

\begin{tabular}{lll}
\hline $\begin{array}{l}\text { Msize: tamaño } \\
\text { del mercado }\end{array}$ & Mercado interno. & Mercado externo. \\
\cline { 2 - 3 } & Índice de tamaño del mercado interno. & $\begin{array}{l}\text { Índice de tamaño del } \\
\text { mercado externo. }\end{array}$ \\
\hline
\end{tabular}

Fuente: Elaboración propia, sobre la base de Foro Económico Mundial, The Global Competitiveness Report 2013-2014, Ginebra, 2014.

Cuadro A1.5

Variables relacionadas con las variables utilizadas en el análisis de componentes principales: factores sistémicos

\begin{tabular}{|c|c|c|c|}
\hline & Transporte & \multicolumn{2}{|l|}{ Electricidad y telefonía } \\
\hline $\begin{array}{l}\text { Infra: aspectos } \\
\text { infraestructurales }\end{array}$ & $\begin{array}{l}\text { Calidad de la infraestructura general, calidad de } \\
\text { las carreteras, calidad de los ferrocarriles, calidad } \\
\text { de los puertos, calidad del transporte aéreo. }\end{array}$ & \multicolumn{2}{|c|}{$\begin{array}{l}\text { Calidad de la oferta de energía, suscripciones } \\
\text { de telefonía móvil y fija. }\end{array}$} \\
\hline $\begin{array}{l}\text { Macro: aspectos } \\
\text { macroeconómicos }\end{array}$ & \multicolumn{3}{|c|}{ Saldo presupuestario del gobierno, ahorro nacional bruto, inflación, deuda pública, calificación de crédito del país. } \\
\hline \multirow{2}{*}{$\begin{array}{l}\text { Social: aspectos } \\
\text { sociales }\end{array}$} & Salud. & \multicolumn{2}{|l|}{ Educación primaria. } \\
\hline & $\begin{array}{l}\text { Impacto comercial de la malaria, incidencia } \\
\text { de la malaria, impacto comercial de la } \\
\text { tuberculosis, incidencia de la tuberculosis, } \\
\text { impacto comercial del sida, prevalencia del } \\
\text { sida, mortalidad infantil, esperanza de vida. }\end{array}$ & \multicolumn{2}{|c|}{$\begin{array}{l}\text { Calidad de la enseñanza primaria, tasa de } \\
\text { matriculación en la educación primaria. }\end{array}$} \\
\hline \multirow[t]{2}{*}{$\begin{array}{l}\text { Train: aspectos } \\
\text { sociales }\end{array}$} & Cantidad de educación. & Calidad de la educación. & $\begin{array}{l}\text { Capacitación en el } \\
\text { lugar de trabajo. }\end{array}$ \\
\hline & $\begin{array}{l}\text { Tasa de matriculación en la enseñanza } \\
\text { secundaria, tasa de matriculación } \\
\text { en la educación terciaria. }\end{array}$ & $\begin{array}{l}\text { Calidad del sistema educativo, } \\
\text { calidad de la enseñanza de } \\
\text { la matemática y las ciencias, } \\
\text { calidad de la gestión escolar. }\end{array}$ & $\begin{array}{l}\text { Disponibilidad local de } \\
\text { investigación especializada } \\
\text { y capacitación, extensión de } \\
\text { la formación del personal. }\end{array}$ \\
\hline \multirow{2}{*}{$\begin{array}{l}\text { Compet: aspectos } \\
\text { legales y normativos }\end{array}$} & Competencia interna. & \multicolumn{2}{|l|}{ Competencia externa. } \\
\hline & $\begin{array}{l}\text { Intensidad de la competencia local, extensión } \\
\text { del dominio del mercado, eficacia de la política } \\
\text { antimonopolística, efecto de la tributación en } \\
\text { los incentivos a la inversión, tasa de impuesto } \\
\text { total, número de trámites necesarios para crear } \\
\text { una empresa, tiempo necesario para crear } \\
\text { una empresa, costos de la política agrícola. }\end{array}$ & \multicolumn{2}{|c|}{$\begin{array}{l}\text { Prevalencia de barreras comerciales, aranceles comerciales, } \\
\text { prevalencia de la propiedad extranjera, impacto comercial } \\
\text { de las normas sobre inversión extranjera directa (IED), } \\
\text { carga de los procedimientos aduaneros, importaciones } \\
\text { como porcentaje del producto interno bruto (PIB). }\end{array}$} \\
\hline \multirow{2}{*}{$\begin{array}{l}\text { Labor: aspectos } \\
\text { sociales }\end{array}$} & Flexibilidad. & \multicolumn{2}{|l|}{ Uso eficiente del talento. } \\
\hline & \multicolumn{3}{|c|}{$\begin{array}{l}\text { Pagos y productividad, dependencia en la gestión profesional, capacidad del país para atraer talento, } \\
\text { capacidad del país para retener talento, participación de la mujer en la fuerza de trabajo. }\end{array}$} \\
\hline
\end{tabular}

Fuente: Elaboración propia, sobre la base de Foro Económico Mundial, The Global Competitiveness Report 2013-2014, Ginebra, 2014. 